Association for Information Systems AIS Electronic Library (AISeL)

Wirtschaftsinformatik Proceedings 2001

Wirtschaftsinformatik

September 2001

\title{
Supply Chain Management: das 'Netzwerk' bestimmt die Logistikprozesse
}

Wolfgang Bosch

IDS Scheer AG, w.bosch@ids-scheer.de

Follow this and additional works at: http://aisel.aisnet.org/wi2001

\section{Recommended Citation}

Bosch, Wolfgang, "Supply Chain Management: das 'Netzwerk' bestimmt die Logistikprozesse" (2001). Wirtschaftsinformatik

Proceedings 2001. 4.

http://aisel.aisnet.org/wi2001/4

This material is brought to you by the Wirtschaftsinformatik at AIS Electronic Library (AISeL). It has been accepted for inclusion in Wirtschaftsinformatik Proceedings 2001 by an authorized administrator of AIS Electronic Library (AISeL). For more information, please contact elibrary@aisnet.org. 
In: Buhl, Hans Ulrich, u.a. (Hg.) 2001. Information Age Economy; 5. Internationale Tagung Wirtschaftsinformatik 2001. Heidelberg: Physica-Verlag

ISBN: 3-7908-1427-X

(C) Physica-Verlag Heidelberg 2001 


\title{
Supply Chain Management: das 'Netzwerk' bestimmt die Logistikprozesse
}

\author{
Wolfgang Bosch \\ IDS Scheer AG
}

\begin{abstract}
Zusammenfassung: Unternehmen sind Teil eines globalen Supply Chain-Netzwerks bestehend aus Kunden, Händlern, Herstellern und Lieferanten. Ihre Geschäftsbeziehungen können die Beteiligten durch netzwerkgerechte Supply ChainProzesse optimieren. Diese Prozesse müssen von den Beteiligten im Netzwerk gemeinsam durch geeignete Werkzeuge gestaltet, implementiert, betrieben und permanent im Hinblick auf die Effizienz überprüft werden. Dies setzt einen offenen Informationsaustausch und Zusammenarbeit (Collaboration) voraus. Bei der Gestaltung der Netzwerkprozesse müssen drei Faktoren besonders berücksicht werden: die Ausrichtung der Supply Chain Prozesse am Kundennutzen, die Realisierung von Synergien durch Outsourcing von (Teil-) Prozessen sowie die Einbindung von Marktplätzen in die Unternehmensstrategie.
\end{abstract}

\section{Unternehmen als Teil eines globalen Netzwerks}

Die Frage nach der Gestaltung einer “optimalen" Supply Chain stellt einen zentralen Aspekt für den Erfolg eines Unternehmens dar. Diese Frage wird auch in der Zukunft aktuell bleiben, da das futuristische Bild, in dem Gegenstände nicht physisch transportiert, sondern nach Bedarf direkt beim Konsumenten durch einen Rechner erzeugt werden, noch in weiter Ferne liegt.

Supply Chain Management oder das effiziente Management von Beziehungen entlang der Wertschöpfungskette bedeutet Realisierung von Wettbewerbsvorteilen in einem zunehmend virtuellen und zugleich transparenten Markt.

Noch vor einigen Jahren stellte die unternehmensweite Gestaltung von Geschäftsprozessen die wesentliche Herausforderung für die Unternehmensführung dar.

Zukünftig, basierend auf der rasanten Entwicklung der Internet- und Mobilfunktechnologie, gilt es, Geschäftsprozesse über die Grenzen der einzelnen Unternehmen hinweg zu gestalten, d.h. die Supply Chain im Sinne eines global funktionierenden Netzwerks auszubauen. Hierbei besteht das Netzwerk aus Kunden, Händlern, Produzenten, Lieferanten und weiteren Partnern, wie z.B. für produktrelevante Dienstleistungen. 
Im nachfolgenden sollen die wichtigsten Merkmale vorgestellt werden, die aus Sicht der IDS Scheer ein erfolgreiches Supply Chain-Netzwerk charakterisieren:

- $\quad$ Kundenfokus,

- $\quad$ Kostenmanagement und

- Collaboration

\section{Kundenfokus}

Der Endkunde und seine Bedürfnisse haben Auswirkung auf die gesamte Logistikkette. Sein Konsumentenverhalten entscheidet über den Erfolg bzw. Misserfolg von Unternehmen, auch wenn sie nicht direkt an den Endkunden liefern.

Was verändert sich beim Endkunden? Er ist in jeder Hinsicht anspruchsvoller als früher. Selbst wenn man direkte Produkteigenschaften einmal ausklammert, gibt es noch genügend Spielfelder, auf dem der Endkunde seinen Anspruch "austoben" kann: er will die Auslieferung "just in time", schneller, billiger und bequemer.

Folgende Trends sind zu beobachten:

- Die Anzahl der Auslieferungen nimmt schnell zu bei gleichzeitiger Reduktion der Menge pro Lieferung.

- Die Auslieferungswünsche werden individueller hinsichtlich Lieferzeit, Verpackung und Teillieferung bei gleichzeitiger Forderung nach billiger (kostenloser) Lieferung.

- Der Kunde erwartet eine schnellere Lieferung bei gleichzeitiger Verkürzung der Vorwarnzeit.

Denkt man diese Trends ein paar Jahre weiter, kommt man schnell zum Schluss, dass der Wettbewerb der Unternehmen bald zum Wettbewerb der logistischen Netzwerke wird.

Der Kunde wird auch zunehmend mobiler. Nokia als Marktführer im weltweiten Mobilfunkgerätemarkt ${ }^{1}$ schätzt, daß im Jahr 2002 weltweit über eine Milliarde Menschen über ein mobiles Telefon verfügen wird. Durch die zunehmende Verschmelzung zwischen der Internet- und der Mobiltechnologie werden im Jahr 2003 mehr mobile Telefone über einen Internet-Anschluß verfügen als PCs ${ }^{2}$.

Die zunehmende Standardisierung der Verfahren in der elektronischen Geschäftsabwicklung, wie bargeldloses Zahlen oder digitale Signatur, machen Internet und

vgl. FAZ, 1./2.6.2001, S. 24

vgl. Nokia’s Business Review 2000, S. 11 
Mobilfunk in Zukunft zum ernstzunehmenden Vertriebskanal: der Kunde kann jederzeit und überall seine Waren und Dienstleistungen erwerben. Diese Entwicklungen müssen Unternehmen in ihrer Strategie berücksichtigen: hier geht es um die Gewinnung von Marktanteilen.

Der zentrale Aspekt des Markterfolgs ist die Ausrichtung der Prozesse am Kundennutzen, der im elektronischen Handel aufgrund der fehlenden emotionalen Bindung zum Kunden noch wichtiger ist als im Ladengeschäft. P(eople)-Business: es gilt über den Kundennutzen die Kundenzufriedenheit, das Kundenvertrauen und die langfristige Kundentreue zu gewinnen. Denn nur ein treuer Kunde wird wiederkommen. Aus der Supply Chain wird im Ergebnis die Customer Value Chain.

Konkret bedeutet die Ausrichtung am Kundennutzen, daß sich die Unternehmen an den Kundenpräferenzen und Einkaufsgewohnheiten ausrichten. Dies bedeutet zum einen, daß der Kunde in die operative Steuerung der Supply Chain eingebunden wird: er entscheidet mit über den Ort, die Zeit der Lieferung sowie die Gestaltung der Leistung. Der Anbieter ist gut beraten, das gegebene Versprechen einzuhalten, termingerecht, preiswert und in geforderter Menge und Qualität zu liefern. Dies setzt einen offenen Informationsaustausch, d.h. eine transparente Supply Chain voraus.

Zum anderen bedeutet dies, daß die Unternehmen Daten aus operativen Systemen extrahieren müssen, um daraus personifizierte Angebote im Sinne eines one-toone Marketing oder nach Kundentypen segmentierte Angebots- und Sortimentspolitik abzuleiten.

Eine weiteres Charakteristikum eines am Kundennutzen ausgerichteten Systems ist, dass Retouren und Reklamationen, z.B. durch Abholservice, Einsatz geeigneter Verpackungssysteme inkl. der erforderlichen Gutschrift, schnell und unbürokratisch abgewickelt werden.

\section{Kostenmanagement}

Die Transparenz am Markt sowie die geringen Zugangsbarrieren zum globalen Markt erhöhen den Druck auf die Unternehmen, nicht nur termingerecht und in geforderter Menge und Qualität, sondern auch preiswert zu liefern.

Die Unternehmen sind nunmehr angehalten, die Kostenstruktur ihrer Wertschöpfungsprozesse zu überprüfen: durch Prozessoptimierung oder durch Outsourcing von (Teil-) Prozessen können positive Kosteneffekte realisiert werden.

Kostenersparnisse lassen sich unter anderem erzielen durch eine

- Reduktion der eigenen Wertschöpfung

- Stärkere Produktstandardisierung 
- Verkürzung von Produktentwicklungszeiten, z.B. durch „Collaborative Engineering“"

- Bildung von Einkaufsgemeinschaften

Beispiele für solche internationale Einkaufsgemeinschaften im Business-to-Business - Bereich sind Covisint (Automobilbereich), Eutilia (Utility) oder ec4ec.com (Anlagenbau).

Nach Gary C. Valade, Vorstand Global Procurement \& Supply von DaimlerChrysler, realisierte der Konzern im letzten Jahr eine Kostensenkung in der Beschaffung von ca. $17 \%$ bei über 500 Teilen und Dienstleistungen und einem Einkaufsvolumen von über $€ 220$ Mio. Das Gesamteinkaufsvolumen beträgt dabei $€ 103,1$ Mrd., was bei einem Umsatz von $€ 147,2$ Mrd. eine eigene Wertschöpfung von ca. $30 \%$ ergibt $^{3}$.

Sollen Synergien über Einkaufgemeinschaften realisiert werden, so muss im Hinblick auf den angestrebten Erfolg und unter Berücksichtigung der gegenwärtigen Marktplatzvielfalt sorgfältig überprüft werden, ob ein eigener Markplatz etabliert werden soll oder ob eine Anbindung an einen bestehenden Marktplatz möglich ist.

Grundsätzlich geht die IDS Scheer davon aus, daß es bei den Markplätzen zu einer starken Konzentration kommen wird.

\section{Collaboration}

Neben dem Kundenfokus und Kostenmanagement stellt die Collaboration das dritte Merkmal eines erfolgreichen Supply Chain-Netzwerks dar.

Die "Collaboration" bezeichnet das gemeinsame Arbeiten auf Basis netzwerkgerechter Prozesse. Diese Prozesse müssen von den Beteiligten im Netzwerk gemeinsam gestaltet, implementiert, betrieben und permanent im Hinblick auf die Effizienz überprüft werden.

Die Vielzahl der zu berücksichtigenden Faktoren wird an einem Praxisbeispiel gezeigt: in einer stark vereinfachten Wertschöpfungskette wird praktisch geprüft, ob ein zusätzlicher Kundenbedarf mit der Menge 30 erfüllbar ist. Der Seminarteilnehmer erlebt dabei persönlich, wie schwierig eine Aussage selbst in einfachsten Modellen "aus dem Kopf" zu treffen ist.

Collaboration erfordert einen offenen Informationsaustausch im Netzwerk und impliziert einen Wandel in der Einstellung hinsichtlich des Umgangs mit unter-

3 vgl. Geschäftsbericht DaimlerChrysler AG 2000, S. 52

"In 2000 für über 220 Mio. Euro eingekauft", Stuttgart/Auburn Hills, Michigan, 22.12.2000, http://www.daimlerchrysler.de/ 
nehmensinternen Informationen, z.B. über Produktions- und Einkaufsmengen oder Absatzpläne. Collaboration ist in dieser Hinsicht ein Synonym für ein transparentes Supply Chain-Netzwerk.

Ein solch transparentes Supply Chain Netzwerk hat z.B. DaimlerChrysler für die Lederausstattung im Automobil aufgebaut. Mit nur drei Kernzahlen pro Fertigungsstufe wird die komplette Wertschöpfungskette mit bis zu 11 Stufen gesteuert.

\section{Zusammenfassung}

Kundenfokus, Kostenmanagement und Collaboration sind die entscheidenden Faktoren für ein effizientes Managment der Supply Chain über Unternehmensgrenzen hinweg. 